\title{
MIG (CXCL9), IP-10 (CXCL10) and I-TAC (CXCL11) concentrations after nasal allergen challenge in patients with allergic rhinitis
}

\author{
Damian Tworek ${ }^{1}$, Piotr Kuna ${ }^{1}$, Wojciech Młynarski², Paweł Górski', Tadeusz Pietras ${ }^{3}$, Adam Antczak ${ }^{3}$
}

1Department of Internal Diseases, Asthma and Allergy, Medical University of Lodz, Poland 2Department of Pediatrics, Oncology, Hematology and Diabetology, Medical University of Lodz, Poland

${ }^{3}$ Department of General and Oncological Pneumonology, Medical University of Lodz, Poland

Submitted: 12 May 2011

Accepted: 24 July 2011

Arch Med Sci 2013; 9, 5: 849-853

DOI: 10.5114 /aoms.2013.37198

Copyright $\odot 2013$ Termedia \& Banach

\author{
Corresponding author: \\ Prof. Adam Antczak \\ Department of Pneumonology \\ and Allergy \\ Medical University of Lodz \\ 22 Kopcinskiego St \\ 90-153 Lodz, Poland \\ Phone: +48 426787505 \\ Fax: +48 426782129 \\ E-mail: \\ adam.antczak@umed.lodz.pl
}

\begin{abstract}
Introduction: The role of monokine induced by interferon- $\gamma$ (IFN- $\gamma$, MIG/CXCL9), IFN- $\gamma$-inducible protein (IP-10/CXCL10), and IFN-inducible T cell $\alpha$ chemoattractant (I-TAC/CXCL11) in allergic inflammation has not been explored in detail in vivo. The aim of the study was to examine the changes in concentrations of MIG/CXCL9, IP-10/CXCL10 and I-TAC/CXCL11 in nasal lavages collected from healthy and allergic subjects during nasal allergen challenge.

Material and methods: Subjects allergic to grass pollen and healthy controls were included. Nasal allergen challenge preceded by placebo administration was performed outside the pollen season. Nasal lavages were collected before and 30 min after application of the placebo and 30 min after allergen administration. Concentrations of chemokines were determined using ELISA.

Results: We observed significantly higher concentrations of IP-10/CXCL10 in allergic patients compared to the healthy subjects before $(354.49 \pm 329.24$ vs. $164.62 \pm 175.94 \mathrm{pg} / \mathrm{ml} ; p=0.036), 30 \mathrm{~min}$ after placebo ( $420.3 \pm 421.28 \mathrm{vs} .246 .88$ $\pm 353.24 \mathrm{pg} / \mathrm{ml} ; p=0.021)$ and $30 \mathrm{~min}$ after allergen administration $(403.28$ \pm 359.29 vs. $162.68 \pm 148.69 \mathrm{pg} / \mathrm{ml} ; p=0.025)$. IP-10/CXCL10 levels did not change 30 min after allergen provocation. In contrast, MIG/CXCL9 levels were similar in both groups before and after placebo. However, a significant rise in MIG/CXCL9 concentration was noted in allergic patients $30 \mathrm{~min}$ after the allergen (138.88 \pm 109.59 vs. $395.8 \pm 301.2 \mathrm{pg} / \mathrm{ml} ; p=0.00026)$. I-TAC/CXCL11 concentrations increased after placebo as well as the allergen in both groups.

Conclusions: IP-10/CXCL10 concentrations are elevated in nasal lavages from allergic patients and this chemokine may play a role in chronic allergic inflammation. MIG/CXCL9 levels increase rapidly after allergen application, which may suggest its role in the early allergic response. Results on I-TAC/CXCL11 concentrations remain inconclusive.
\end{abstract}

Key words: IP-10, MIG, I-TAC, allergic inflammation, rhinitis.

\section{Introduction}

Monokine induced by interferon- $\gamma$ (IFN- $\gamma$; MIG/CXCL9), IFN- $\gamma$-inducible protein (IP-10/CXCL10), and IFN-inducible T cell $\alpha$ chemoattractant (I-TAC/ CXCL11) are chemokines traditionally considered as chemoattractants associated with Th1-type response and inflammation. However, there is some evidence showing that they are involved in development of allergic inflamma- 
tion. Many papers have been published on the role of these chemokines in skin allergic diseases but the knowledge of their function in respiratory allergic diseases is scanty and mainly refers to in vitro and animal studies. It is known that MIG/CXCL9 and IP10/CXCL10 may be produced by eosinophils after stimulation with INF- $\gamma$ but this effect is suppressed by Th2-cytokine interleukin (IL)-4 [1-4]. This may indicate that MIG/CXCL9 and IP-10/CXCL10 may take part in downregulation of allergic inflammation. However, there are some reports showing that at least IP-10/CXCL10 and MIG/CXCL9 may be important factors playing a role in development of the inflammatory state in asthma and asthma exacerbations [4].

In this pilot study we examined basal concentrations of MIG/CXCL9, IP-10/CXCL10 and I-TAC/ CXCL11 in nasal lavages collected from healthy and allergic subjects outside of the pollen season as well as the potential effect of application of the relevant allergen on the levels of the chemokines during the early phase of an allergic reaction.

\section{Material and methods}

\section{Study group}

Consecutive patients with allergic rhinoconjunctivitis due to grass pollen allergy and healthy volunteers were included. Patients were considered allergic when they had a history of symptoms of rhinoconjunctivitis during at least two grass pollen seasons (May-July) and positive results of skin prick tests only with grasses and specific immunoglobulin (Ig) E class $>$ II. The control group consisted of healthy volunteers with similar demographic characteristics to those of allergic subjects.

Subjects with a history of perennial symptoms, nasal septal deviation, nasal polyps, chronic sinusitis, tobacco smoking (current and former) and infection of the lower and/or upper respiratory tract during 4 weeks preceding the challenge were omitted from the study.

Based on the inclusion/exclusion criteria $24 \mathrm{pa}$ tients with allergy to grass pollen (16 males, mean age $24.9 \pm 7.3$ years; duration of rhinitis $4.9 \pm 3.7$ years) and 24 healthy subjects from the control group took part in the study (16 males, mean age $27.1 \pm 11.4$ years). Mean slgE serum level in allergic subjects was $11.08 \pm 8.84 \mathrm{kU} / \mathrm{l}$.

The protocol of this study was approved by the Ethical Committee of the Medical University of Lodz and all participants gave their written informed consent.

\section{Study design}

Before any procedure the subjects rested for $30 \mathrm{~min}$ to accommodate to the room temperature. First, they filled in a visual analogue scale and they had peak nasal inspiratory flow (PNIF) measured and at the end nasal lavages were collected. Sub- sequently control solution was administered and after 30 min all the procedures were repeated. Next, the allergen was administered and after 30 min the procedures were performed once again.

\section{Skin prick tests}

All patients had the skin prick tests performed with common aeroallergens. Tested aeroallergens were Dermatophagoides pteronyssinus, Dermatophagoides farinae, grasses, birch, hazel, alder, mugwort, cat, dog, Alternaria tenuis and Cladosporium herbarum (Allergopharma, Reinbeck, Germany). Histamine 1.7 mg/ml (Allergopharma, Reinbeck, Germany) and standard glycerol saline solution (Allergopharma, Reinbeck, Germany) were used as a positive and negative control, respectively. Wheal diameter $\geq 3 \mathrm{~mm}$ was considered as a positive result of the test.

\section{Specific IgE}

Serum specific IgE levels were determined using the ELISA method with a commercially available TR kit (Allergopharma, Reinbeck, Germany).

\section{Nasal allergen challenge}

Single blind nasal allergen challenge was performed using standardized grass pollen extract (Allergopharma, Reinbeck, Germany). Diluent for the allergen was used as the control solution.

Two puffs of allergen solution (5000 SBU/ml) were administered into one nostril, after previous application of placebo (control solution).

The use of the following medications was prohibited before the nasal challenge: nasal and systemic glucocorticosteroids for 28 days, oral and nasal antihistamines, antileukotrienes and oral preparations of pseudoephedrine for 14 days, nasal $\alpha$-agonists and non-steroidal anti-inflammatory drugs for 7 days.

Nasal challenge was performed outside the pollen season (October-March).

\section{Visual analogue scale}

Severity of clinical symptoms (rhinorrhea, nasal itching, eye itching, sneezing, blockage, postnasal drip) was described by patients before and after the challenge with a visual analogue scale (VAS) (12) and then transferred to numerical values $(0-\mathrm{min}$ imum, 100 - maximum points for one symptom, resulting in 600 points in total) for further analyses.

\section{Nasal lavages}

Nasal lavages were collected according to the method described by Greiff et al. in time points specified above [5]. A centrifugation (10 $\mathrm{min}$ at $0.08 \mathrm{~g}$, $4^{\circ} \mathrm{C}$ ) of saline washings separated the cell pellet and 
supernatant. The supernatant was immediately frozen $\left(-70^{\circ} \mathrm{C}\right)$ for further determination of $\beta$-tryptase levels. The obtained sediment was washed in a sterile phosphate buffered saline (PBS, Sigma) and then suspended in $1.0 \mathrm{ml}$ of RPMI 1640 (Sigma). Differential cell counts were performed on slides stained by the May-Grunwald-Giemsa method. A minimum of 400 cells was counted per smear to enable a differential cell count to be made for each specimen. Cells were classified according to their morphology as neutrophils, eosinophils, basophils, lymphocytes and squamous cells.

\section{$\beta$-Tryptase}

$\beta$-Tryptase concentrations in nasal lavages were measured using UniCAP Tryptase Fluoroenzyme immunoassay (Pharmacia, Uppsala, Sweden) with a detection limit of $1.0 \mu \mathrm{gl} / \mathrm{l}$. For statistical analysis, values below $1.0 \mu \mathrm{g} / \mathrm{l}$ were considered as $0 \mu \mathrm{g} / \mathrm{l}$.

\section{PNIF measurements}

PNIF measurements were performed under supervision using an In-Check portable nasal inspiratory flow meter (Clement Clarke International, Harlow, United Kingdom). The subjects were instructed on how to use the device correctly to obtain reliable recordings. Three measurements were recorded for each subject. The best of three maneuvers was used for further calculations. The measurement was repeated when incorrect technique was noticed.

\section{Chemokine assays}

MIG/CXCL9, IP-10/CXCL10 and I-TAC/CXCL11 levels in nasal lavages were determined using commercially available ELISA kits (R\&D Systems, Minneapolis, USA) according to the manufacturer's instructions.

\section{Statistical analysis}

Statistical analysis was performed using Statistica 8.0 (StatSoft, Tulsa, USA). The results are presented as mean \pm standard deviation. Data were tested for normal distribution using Shapiro-Wilk test. Depending on the distribution of the variables, comparisons were performed using paired Student's t-test and Wilcoxon's test for paired variables and unpaired Student's t-test and Mann-Whitney $U$-test for unpaired variables. Correlations were analyzed using Spearman $R$ coefficient.

\section{Results}

\section{Rhinitis symptoms assessed with VAS}

There was no significant change in nasal symptoms after application of the control solution in aller- gic subjects (17.4 \pm 20.94 at baseline vs. $27.3 \pm 33.34$ points $30 \mathrm{~min}$ after placebo; $p=0.31$ ). There was a significant increase in rhinitis symptoms in allergic patients after the allergen administration (27.3 \pm 33.34 points after placebo vs. $152.74 \pm 81.95$ points 30 min after the allergen; $p<0.001$ ). No changes in rhinitis symptoms were observed after application of either the control solution ( $23.72 \pm 29.34$ points at baseline vs. $35.36 \pm 46.84$ points 30 min after placebo; $p=0.86$ ) or the allergen (35.36 \pm 46.84 points after placebo vs. $30.63 \pm 39.0$ points $30 \mathrm{~min}$ after the allergen; $p=0.98$ ) in the control group.

\section{PNIF}

There was no difference in PNIF between healthy controls and allergic patients before (147.2 \pm 47.7 $\mathrm{ml} / \mathrm{s}$ vs. $149.5 \pm 36.9 \mathrm{ml} / \mathrm{s} ; p=0.95$, respectively) or after $(141.3 \pm 46.3 \mathrm{ml} / \mathrm{s}$ vs. $145.2 \pm 39.1 \mathrm{ml} / \mathrm{s} ; p=0.69$, respectively) application of the control solution. Moreover, administration of the control solution did not influence PNIF values in any group. Allergen challenge led to a fall in PNIF in the allergic group, on average by $44.6 \pm 35.0 \%$, to $96.7 \pm 42.8 \mathrm{ml} / \mathrm{s}$ $(p=0.000004)$, which was not observed in healthy subjects (146.4 $\pm 39.0 \mathrm{ml} / \mathrm{s} ; p=0.6794)$.

\section{$\beta$-Tryptase}

There were no differences in $\beta$-tryptase concentrations in nasal lavages in the control group at successive time points. In the group of allergic patients a significant rise in $\beta$-tryptase levels was observed after administration of the allergen ( $0.067 \pm 0.32$ vs. $3.66 \pm 3.69 \mu \mathrm{g} / \mathrm{ml} ; p<0.001)$.

\section{Nasal lavages cell count}

No significant differences in nasal lavages cell count between healthy and allergic subjects were found before and 30 min after administration of control solution. Administration of the allergen did not influence nasal lavages cell count in the control group, but in allergic patients we observed a significant fall in the percentage of squamous cells $(71.7 \pm 16.5 \% \mathrm{vs}$. $56.6 \pm 22.7 \%$; $p=0.0049$ ) and a rise in the percentage of neutrophils and percentage of eosinophils (13.7 $\pm 11.8 \%$ vs. $20.3 \pm 15.0 \% ; p=0.034$ and $7.6 \pm 9.4 \%$ vs. $15.1 \pm 14.1 \% ; p=0.012375$, respectively). There was a significant difference between the groups in percentage of squamous cells ( $p=0.007592)$, percentage of neutrophils $(p=0.042339)$ and percentage of eosinophils $(p=0.007592)$ after allergen challenge.

\section{Chemokine concentrations during allergen challenge}

Concentrations of IP-10/CXCL10 did not change significantly after administration of placebo or the allergen. However, at all time points significantly 


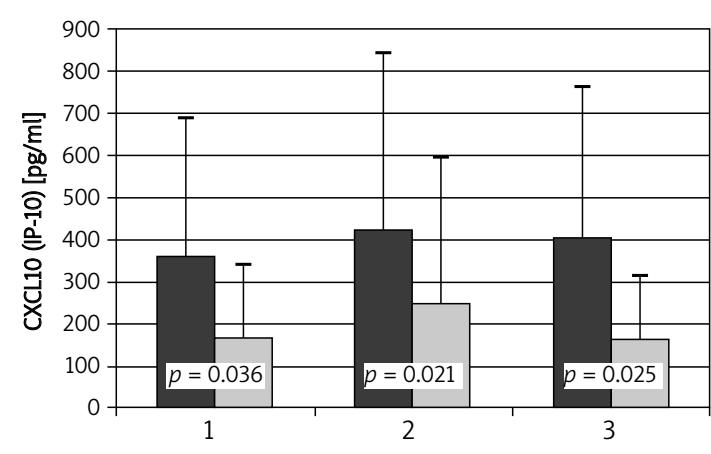

$\square$ Allergic

Non-allergic

Figure 1. Changes in IP-10/CXCL10 concentrations during allergen challenge. 1 - before administration of negative control solution; 2 - after administration of negative control solution/before administration of the allergen; 3 - after administration of the allergen

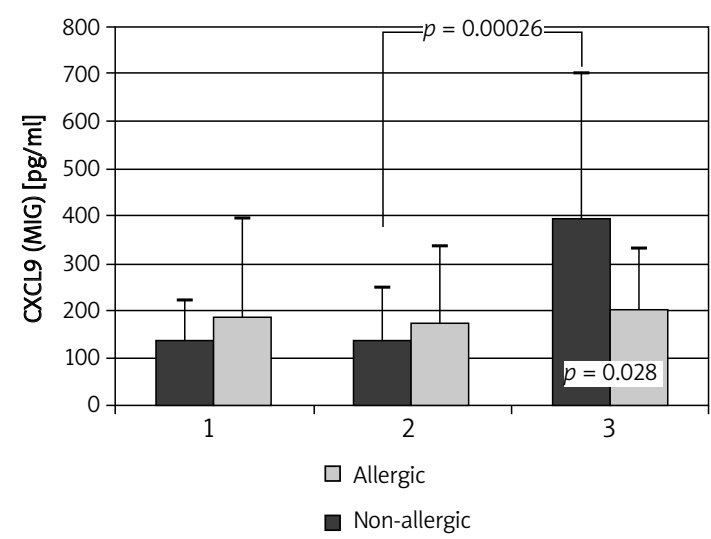

Figure 2. Changes in MIG/CXCL9 concentrations during allergen challenge. 1 - before administration of negative control solution; 2 - after administration of negative control solution/before administration of the allergen; 3 - after administration of the allergen

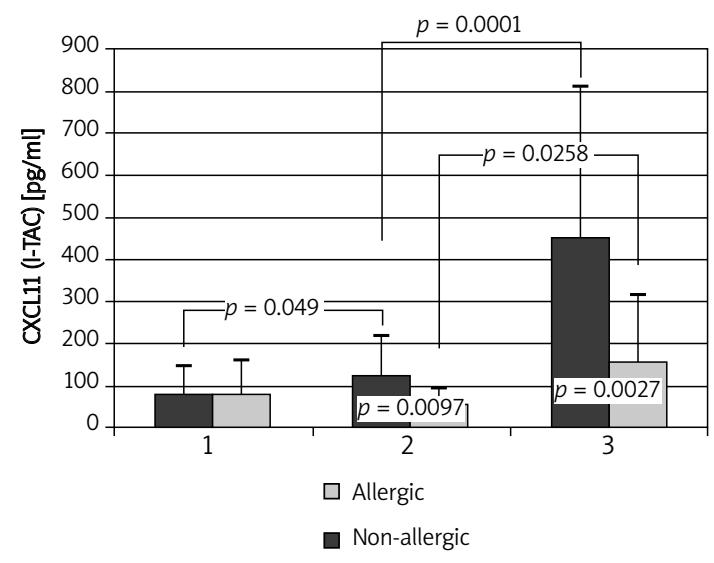

Figure 3. Changes in I-TAC/CXCL11 concentrations during allergen challenge. 1 - before administration of negative control solution; 2 - after administration of negative control solution/before administration of the allergen; 3 - after administration of the allergen higher concentrations of IP-10/CXCL10 in nasal lavages were observed in the allergic group compared to healthy controls (354.49 \pm 329.24 vs. 164.62 $\pm 175.94 ; p=0.036 ; 420.3 \pm 421.28$ vs. 246.88 $\pm 353.24 ; p=0.021$ and $403.28 \pm 359.29$ vs. 162.68 $\pm 148.69 ; p=0.025$, respectively) (Figure 1).

MIG/CXCL9 concentrations were similar in both groups at baseline and 30 min after application of control solution. Administration of the allergen led to a significant increase in MIG/CXCL9 levels in allergic subjects (138.88 \pm 109.59 vs. $395.8 \pm 301.2$ $\mathrm{pg} / \mathrm{ml} ; p<0.001$ ) (Figure 2).

I-TAC/CXCL11 levels increased after administration of placebo in allergic patients (77.84 \pm 67.45 vs. $124.72 \pm 93.2 \mathrm{pg} / \mathrm{ml} ; p=0.049)$. After administration of the allergen a further increase in I-TAC concentrations to $450.78 \pm 358.15 \mathrm{pg} / \mathrm{ml}(p<0.0001)$ was observed in allergic patients, but in the control group the chemokine levels rose as well (53.01 \pm 36.65 vs. $156.99 \pm 157.58 \mathrm{pg} / \mathrm{ml} ; p=0.026$ ) (Figure 3).

\section{Correlations between chemokines and symptoms of rhinitis, PNIF and nasal lavages cell count and $\beta$-tryptase}

We did not find any correlation between total symptoms score and MIG/CXCL9 and IP-10/CXCL10 after the challenge in allergic patients $(r=0.22$; $p=0.33$ and $r=0.056 ; p=0.8$, respectively). In addition, the concentrations of the chemokines did not correlate with percentage of change in PNIF $(r=-0.18 ; p=0.42$ and $r=0.03 ; p=0.89$; for MIG/ CXCL9 and IP-10/CXCL10, respectively).

MIG/CXCL9 and IP-10/CXCL10 levels did not correlate with the percentage of eosinophils $(r=0.19$; $p=0.41$ and $r=0.12 ; p=0.59$, respectively) or lymphocytes $(r=-0.001 ; p=0.99$ and $r=0.32 ; p=0.15$, respectively) in nasal lavages from allergic patients after allergen administration.

$\beta$-tryptase levels in nasal lavages did not correlate with MIG/CXCL9 $(r=-0.012 ; p=0.96)$ or IP-10 $(r=-0.023 ; p=0.92)$ after the challenge.

I-TAC/CXCL11 was omitted from the analysis as a significant rise in its levels was also observed in the control group.

\section{Discussion}

In this pilot study we found that MIG/CXCL9 and IP-10/CXCL10 may play a role in the development of nasal allergic inflammation. The presented data focused on the early phase of the allergic reaction, which is the main limitation of this study. Nevertheless, we were able to observe the changes in MIG/CXCL9 concentrations 30 min after the application of the allergen and higher baseline levels of IP-10/CXCL10 in allergic subjects compared to healthy controls outside the pollen season.

Miotto et al. found that IP-10/CXCL10 is overexpressed in asthmatic lower airways, but to a less- 
er extent than in subjects with sarcoidosis, which is mainly a Th1 mediated disease [6]. Similarly, IP-10/CXCL10 as well as MIG/CXCL9 and I-TAC/ CXCL11 were detected in higher concentrations in bronchoalveolar fluid of asthmatic children when compared to control subjects [7]. Moreover, IP-10/ CXCL10 and MIG/CXCL9 plasma levels are higher during an acute asthma exacerbation than during a subsequent convalescent period or when compared to patients with stable disease [8]. The important role of IP-10/CXCL10 in development of airway hyperresponsiveness and Th2-type allergic inflammation was proven in a murine model of asthma [9]. Our results show that in nasal lavages of patients allergic to grass pollen IP-10/CXCL10 levels are overexpressed outside the pollen season, which may indicate subclinical inflammation in these subjects despite the lack of exposure to the relevant allergen. This hypothesis is supported by previous findings of our group showing increased levels of 8-isoprostane in nasal lavages obtained outside the pollen season from patients with seasonal allergic rhinitis [10]. Our study indicates that IP-10/CXCL10 levels do not rise during the early phase reaction; however, it was previously noted that concentrations of this chemokine significantly increase $20 \mathrm{~h}$ after intrabronchial administration of the allergen in bronchoalveolar fluid collected at the challenged site [11].

MIG/CXCL9 concentrations were similar in allergic patients and healthy controls at baseline and after administration of control solution but a rapid increase in its levels in nasal lavages was observed 30 min after the challenge with grass pollen. It was previously shown in an animal model of asthma that allergen-induced MIG/CXCL9 acts as a specific and natural inhibitor of eosinophils' recruitment and CCR3-mediated functional response of these cells [12]. Therefore the increase in MIG/CXCL9 concentrations after administration of allergen may be explained as part of the negative feedback leading to suppression of the inflammatory response to the allergen.

It is difficult to determine the influence of the allergen on levels of I-TAC/CXCL11 as it seems that administration of the control solution itself leads to increase in the chemokine concentrations, even in healthy subjects. We speculate that it may be caused by phenol used as the preservative for the allergen solution. Nevertheless, it is worth mentioning that we observed a much more pronounced increase in I-TAC/CXCL11 levels in nasal lavages after allergen administration in allergic subjects, which may indicate that I-TAC/CXCL11 concentrations also rise in response to exposure to allergens.

In conclusion, in this pilot study we showed that Th1 chemokines MIG/CXCL9, IP-10/CXCL10 and probably I-TAC/CXCL11 take part in development of allergic inflammation of the nose and subclinical inflammation persists outside the pollen season in patients with seasonal allergic rhinitis. More studies are necessary to analyze the kinetics of changes in expression of these chemokines in nasal mucosa in regard to inflammatory cell infiltration and other markers of inflammation.

\section{References}

1. Meller S, Lauerma Al, Kopp FM, et al. Chemokine responses distinguish chemical-induced allergic from irritant skin inflammation: memory T cells make the difference. J Allergy Clin Immunol 2007; 119: 1470-80.

2. Flier J, Boorsma DM, Bruynzeel DP, et al. The CXCR3 activating chemokines IP-10, Mig, and IP-9 are expressed in allergic but not in irritant patch test reactions. J Invest Dermatol 1999; 113: 574-8.

3. Flier J, Boorsma DM, van Beek PJ, et al. Differential expression of CXCR3 targeting chemokines CXCL10, CXCL9, and CXCL11 in different types of skin inflammation. J Pathol 2001; 194: 398-405.

4. Dajotoy T, Andersson P, Bjartell A, Löfdahl CG, Tapper H, Egesten A. Human eosinophils produce the T cellattracting chemokines MIG and IP-10 upon stimulation with IFN-gamma. J Leukoc Biol 2004; 76: 685-91.

5. Greiff L, Meyer P, Svensson C, Persson CG, Andersson M. The "nasal pool"-device for challenge and lavage of the nasal mucosa in children: histamine-induced plasma exudation responses. Pediatr Allergy Immunol 1997; 8: 137-42.

6. Miotto D, Christodoulopoulos P, Olivenstein R, et al. Expression of IFN-gamma-inducible protein; monocyte chemotactic proteins 1, 3, and 4; and eotaxin in TH1- and TH2-mediated lung diseases. J Allergy Clin Immunol 2001; 107: 664-70.

7. Hartl D, Griese M, Nicolai T, et al. Pulmonary chemokines and their receptors differentiate children with asthma and chronic cough. J Allergy Clin Immunol 2005; 115: 728-36.

8. Lai ST, Hung CH, Hua YM, Hsu SH, Jong YJ, Suen JL. T-helper 1-related chemokines in the exacerbation of childhood asthma. Pediatr Int 2008; 50: 99-102.

9. Medoff BD, Sauty A, Tager AM, et al. IFN-gammainducible protein 10 (CXCL10) contributes to airway hyperreactivity and airway inflammation in a mouse model of asthma. J Immunol 2002; 168: 5278-86.

10. Antczak A, Kuźmińska B, Górski P. 8-isoprostane levels are elevated in nasal lavages from asthmatic patients with allergic rhinitis. Allergy 2003; 58 (suppl. 74): 74.

11. Bochner BS, Hudson SA, Xiao HQ, Liu MC. Release of both CCR4-active and CXCR3-active chemokines during human allergic pulmonary late-phase reactions. J Allergy Clin Immunol 2003; 112: 930-4.

12. Fulkerson PC, Zimmermann N, Brandt EB, et al. Negative regulation of eosinophil recruitment to the lung by the chemokine monokine induced by IFN-gamma (Mig, CXCL9). Proc Natl Acad Sci U S A 2004; 101: 1987-92. 